\title{
ESTIMATORS AND BOOTSTRAP CONFIDENCE \\ INTERVALS FOR RUIN PROBABILITIES
}

\author{
CHRISTIAN HipP \\ University of Hamburg, FRG
}

\begin{abstract}
For the infinite time ruin probability in the classical risk process, efficient estimators are proposed in cases in which the claim amount distribution is unknown. Confidence intervals are computed which are based on normal approximations or on the bootstrap method. The procedures are checked in a MonteCarlo study.
\end{abstract}

\section{KEYWORDS}

Infinite time ruin probabilities; nonparametric estimators; confidence intervals; bootstrap method.

\section{INTRODUCTION AND SUMMARY}

\subsection{Ruin probabilities as a measure of risk}

We consider the classical risk process

$$
R(t)=x+\mathrm{ct}-S(t), \quad t \geq 0
$$

with nonnegative initial surplus $x$, positive premium rate $c$, and a claims process $S(t), t \geq 0$, which is a compound Poisson process with positive intensity $\lambda$ and claim amount distribution $Q$. When all parameters $x, c, \lambda$, and $Q$ of the risk process are given, then we can determine the infinite time ruin probability

$$
\psi(x)=\mathbb{P}\{R(t)<0 \text { for some } t>0\}
$$

In the author's opinion, the ruin probability $\psi(x)$ is a measure for the "risk" contained in the process $R(t), t \geq 0$. It should be used to evaluate decisions concerning the risk business. To give an example we assume that we can buy an XL-reinsurance cover with priority $M>0$ and reinsurance premium rate $0<c_{R}<c$. Let $\psi_{R}(x)$ be the ruin probability of the risk process with reinsurance. The parameters of the new risk process are $x, c-c_{R}, \lambda$, and $Q_{R}$ given by

$$
Q_{R}(A)=Q(A \cap(0, M))+1_{A}(M) Q((M, \infty)) .
$$

If $\psi_{R}(x)<\psi(x)$ then the reinsurance cover reduces the "risk" contained in the risk process. So it is reasonable to pay the premium rate $c_{R}$ for the reinsurance cover described above. If, on the other hand, $\psi(x)<\psi_{R}(x)$, then we do better without the above reinsurance cover. 


\subsection{Estimation of ruin probabilities}

In practical applications the parameters $\lambda$ and $Q$ will not be given but have to be estimated with available data. Consider, e.g., the situation that $\lambda$ is known but $Q$ is not. Suppose we are given observations $x_{1}, \ldots, x_{n}$ which are realizations of random variables $X_{1}, \ldots, X_{n}$, these being independent with distribution $Q$. Our nonparametric estimator will be based on the empirical distribution $Q_{n}^{\bar{x}}$ of the observations $\underline{x}=\left(x_{1}, \ldots, x_{n}\right)$. The distribution $Q_{n}^{\bar{x}}$ is discrete and has point probabilities

$$
Q_{n}^{\bar{x}}\{y\}=\frac{1}{n} \#\left\{1 \leq i \leq n: x_{i}=y\right\}, \quad y \in \mathbb{R}
$$

(The symbol \# A denotes the number of elements in the set A). Our nonparametric estimator $\psi_{n}(x)$ is the ruin probability of the risk process with parameters $x, c, \lambda$, and $Q_{n}^{\bar{x}}$. The estimator $\psi_{n}(x)$ is asymptotically normal and efficient, its asymptotic variance is given below. The finite sample behaviour of $\psi_{n}(x)$ is investigated in a Monte Carlo study for sample sizes $n=20$ and $n=100$. Similar nonparametric estimators are proposed for cases in which other or less parameters are known. In order to define these estimators we assume that only positive claims are possible,

$$
Q(0, \infty)=1
$$

that $Q$ is non-degenerate, and the risk process $R(t), t \geq 0$, has a positive safety loading, i.e. $Q$ has a finite mean $\mu=\int y Q(d y)$, and the premium rate $c$ is larger than the average claim amount per unit time,

$$
c>\lambda \mu \text {. }
$$

If (2) does not hold, then $\psi(x)=1$ for all $x$. If (2) is true, then $\psi(0)=\lambda \mu / c<1$ and $\psi(x)$ can be computed according to

$$
\psi(x)=\sum_{k=1}^{\infty}(1-\psi(0)) \psi(0)^{k} \mathrm{IH}^{* k}(x, \infty) .
$$

Here, $* k$ is the $k$-fold convolution, and IH is the distribution of the first ascending ladder height with density

$$
y \rightarrow \mu^{-1} Q(y, \infty) 1_{(0, \infty)}(y)
$$

(see Bühlmann (1970), 6.2.6, or TAylor (1985), p. 75, (3.1.1), or Feller (1966), p. $379,(2.6))$. We shall consider the following four cases:

1) $\lambda$ known, $Q$ unknown; this is the case described above. $\psi_{n}^{(1)}$ is the ruin probability of the risk process with parameter $x, c, \lambda$, and $Q_{n}^{\bar{x}}$.

2) $\lambda \mu$ known, $Q$ unknown; $\psi_{n}^{(2)}$ is given by

$$
\psi_{n}^{(2)}(x)=\sum_{k=1}^{\infty}(1-\psi(0)) \psi(0)^{k} H_{n}^{* k}(x, \infty)
$$


where $H_{n}$ is the measure with density

$$
y \rightarrow \frac{1}{\bar{x}_{n}} Q_{n}^{\bar{x}}(y, \infty) 1_{(0, \infty)}(y)
$$

and $\bar{x}_{n}=\frac{1}{n} \sum_{i=1}^{n} x_{i}$ is the arithmetic mean of all observations.

3) $\lambda, \mu$ known, $Q$ unknown; $\psi_{n}^{(3)}$ is given by

$$
\psi_{n}^{(3)}(x)=\sum_{k=1}^{\infty}(1-\psi(0)) \psi(0)^{k} G_{n}^{* k}(x, \infty)
$$

where $G_{n}$ has density

$$
y \rightarrow \frac{1}{\mu} P_{n}^{\bar{x}}(y, \infty), \quad y>0
$$

and $P_{n}^{\bar{x}}$ is the discrete probability measure with point probabilities

$$
P_{n}^{\bar{x}}\left\{x_{i}\right\}=\frac{1}{n}\left(1-\left(x_{i}-\bar{x}_{n}\right)\left(\bar{x}_{n}-\mu\right) / s^{2}\right)
$$

where

$$
s^{2}=\frac{1}{n} \sum_{j=1}^{n}\left(x_{j}-\bar{x}_{n}\right)^{2}
$$

is the sample variance. The factor $1 / n$ has to be replaced by $\#\left\{j: x_{j}=x_{i}\right\} / n$ if there are multiplicities in the $x$ 's. The numbers in (8) add to 1 , and they are nonnegative with high probability.

4) $\lambda$ unknown, $Q$ unknown; for this case we assume that we have observations from a risk process in a time interval $(0, T)$. Let $N(T)$ be the number of observed claims and $X_{1}, \ldots, X_{N(T)}$ the corresponding claim amounts. The estimator $\psi_{T}^{(4)}$ is the ruin probability of the risk process with parameters $x, c, \hat{\lambda}=N(T) / T$, and $\hat{Q}=$ empirical distribution of $X_{1}, \ldots, X_{N(T)}$.

O course case 4$)$ is the standard situation in practical application. However, knowledge of $\lambda$ and/or $\mu$ can reduce the statistical error substantially, and for these parameters extra data are frequently available (from other companies with similar portfolios or from one of the insurance organizations).

All estimators are asymptotically normal, and their asymptotic variances $\sigma^{(1)}$, $\sigma^{(2)}, \sigma^{(3)}, \sigma^{(4)}$ satisfy the relation

$$
\sigma^{(3)} \leq \sigma^{(2)} \leq \sigma^{(1)} \leq \sigma^{(4)}
$$

This indicates that estimation of $\psi(x)$ seems to be simpler in case 2) than in case 1 ). 


\subsection{Measuring risk with estimated ruin probabilities}

The evaluation of possible decisions in a risk business can be based on estimated ruin probabilities. However, if $\psi_{n}(x)$ is the estimator for the ruin probability $\psi(x)$ without reinsurance and if $\psi_{n}^{(R)}(x)$ is the estimator for the ruin probability $\psi_{R}(x)$ with reinsurance, then the relation

$$
\psi_{n}^{(R)}(x)<\psi_{n}(x)
$$

does not yield the conclusion $\psi_{R}(x)<\psi(x)$; both sides of relation (10) contain a statistical error which, in the worst case, leads to (10) even if the relation $\psi(x)>\psi_{R}(x)$ holds. A possible solution to this problem is the concept of confidence intervals. For prescribed $a$ near zero one can construct a confidence interval $C_{n}$ for $\psi(x)-\psi_{R}(x)$ with

$$
\lim _{n} \operatorname{IP}\left\{\psi(x)-\psi_{R}(x) \in C_{n}\right\}=1-a
$$

If $C_{n}$ lies in the positive half axis, then we conclude that $\psi(x)>\psi_{R}(x)$. If $C_{n}$ lies in the negative half axis, then our conclusion will be $\psi(x)<\psi_{R}(x)$. If, finally, $0 \in C_{n}$, then no conclusion will be possible. With this procedure, for large $n$ the error probability will not exceed $a$, i.e.

$$
\lim \sup _{n} \mathbb{I}\{\text { wrong conclusion }\} \leq a .
$$

The existence of reasonable confidence intervals for $\psi(x)-\psi_{R}(x)$ (not containing zero) is not self evident. The quantities $\psi(x)$ and $\psi_{R}(x)$ are very small and might have the same order of magnitude as the statistical error. In order to investigate this problem we shall restrict consideration to a confidence interval for $\psi(x)$. A possible confidence interval for $\psi(x)$ is the one which is based on the asymptotic normality of our estimators (see 2.2). In a Monte-Carlo study we observe that for sample size $n=20$ and $n=100$ the normal approximation for the distribution of our estimators is rather bad, especially when the claim amount distribution $Q$ has a large coefficient of variation

$$
\tau=\frac{1}{\mu}\left(\int(y-\mu)^{2} Q(d y)\right)^{1 / 2}
$$

The variance of the approximating normal distribution is too large, and consequently the confidence intervals based on the normal approximation are too large. Furthermore, the distribution of $\psi_{n}(x)$ is usually not symmetric about $\psi(x)$ but skew to the left. Hence we cannot expect that confidence intervals which are symmetric about $\psi_{n}(x)$ will perform well. 


\subsection{Bootstrap confidence intervals}

In order to get smaller confidence intervals we used the bootstrap method. The construction of these confidence intervals will be described in case 1) for the sample size $n=20$. Let $X_{1}, \ldots, X_{20}$ be the observations, and let $Q_{20}^{\bar{x}}$ be the empirical distribution of these observations. Generate 100 samples $X^{(1)}, \ldots, X^{(100)}$ of size 20 , i.e. 2000 independent random numbers with distribution $Q_{20}^{\bar{x}}$, grouped in 100 samples of size 20 each. For $i=1, \ldots, 100$ compute the estimator $\psi_{n}(x)$ for the set of observations $X^{(i)}$. We obtain 100 values $\psi_{n, i}(x)$, and we write $U(\underline{x})$ for the empirical distribution of these values. Our confidence interval is the shortest interval $I$ satisfying $U(\underline{x})(I) \geq 1-a$.

The bootstrap confidence intervals are small, and they are not symmetric around $\psi(x)$ in general. In a small Monte-Carlo study with sample size 20, 100 bootstrap resamplings per trial, and 100 Monte-Carlo trials, it turned out, however, that the level of the bootstrap confidence intervals is considerably smaller than $1-a$. The same bad behaviour occured with 200 instead of 100 bootstrap resamplings per trial. On the other hand, the small number of 100 Monte-Carlo trials does not given us precise information on the actual level of our confidence sets. A larger scale Monte-Carlo study with 1000 or more trials is a computer time consuming task: for 1000 trials we need $2 \times 10^{6}$ random numbers and $10^{5}$ computions of $\psi_{n}(x)$.

For sample size 100 the performance of the bootstrap confidence intervals was much better: the level $90 \%$ confidence intervals had an actual covering probability between $87 \%$ and $94 \%$. The average size of the intervals ranges from 0.0002 (in case with $\psi(x)=0.0025)$ to 0.03 (with $\psi(x)=0.032$ ). This indicates that our bootstrap confidence intervals can be used for the evaluation of possible decisions, provided the sample size is not too small.

\subsection{A simple bootstrap selection rule}

Combining the bootstrap with the above decision rule for " $\psi(x)<\psi_{R}(x)$ or not" we obtain the following simple method: Resample $M$ times, say, and compute the estimators $\psi_{n}(x)$ and $\psi_{n}^{(R)}(x)$ for each bootstrap sample. If $\psi_{n}(x)<\psi_{n}^{(R)}(x)$ for at least $(1-a) M$ bootstrap samples, then we conclude that $\psi(x)<\psi_{R}(x)$. If $\psi_{n}(x)>\psi_{n}^{(R)}(x)$ for at least $(1-\alpha) M$ bootstrap samples, then our conclusion is $\psi(x)>\psi_{R}(x)$. If, finally, both conditions are not satisfied, then no conclusion is possible. This method can easily be generalized to more than two alternatives.

Section 2 contains the theoretical results. The findings of our Monte-Carlo simulations are summarized in section 3 .

\subsection{Reference to earlier papers}

In the framework of ruin theory, statistical estimators were first used by GRANDELL (1979). He constructed estimators for the adjustment coefficient $R$ of the 
risk process in the case $\lambda, Q$ unknown. The adjustment coefficient yields the CRAMÉR upper bound for $\psi(x)$,

$$
\psi(x) \leq \exp (-R x)
$$

and the asymptotic behavior of $\psi(x)$ for $x$ large,

$$
\psi(x) \approx C \exp (-R x), \quad C>0 .
$$

Apparently, GRANDELL's results can not be used for the construction of twosided confidence intervals for $\psi(x)$.

Different nonparametric estimators for $\psi(x)$ have been proposed by FreEs (1986). His estimators are based on the sample reuse concept: From the given data set a new risk process is constructed, and the ruin probability of the new risk process is computed with Monte-Carlo methods. The performance of these estimators is rather bad: the root mean squared error in a Monte-Carlo study has the same order of magnitude as $\psi(x)$ for sample sizes $n=25,50,100$, 150, 200 (see Frees (1986), p.S. 87, table 1). This is perhaps due to the fact that FREES' estimators do not only contain a statistical error but also a simulation error.

\section{THE RESULTS}

\subsection{Consistency}

We shall first state some large sample properties of the proposed estimators.

Lemma: Fix $x, c, \lambda>0$ and a claim amount distribution $Q$ for which (2) and (3) hold. Then for $i=1,2,3$

$$
\lim _{n} \psi_{n}^{(i)}(x)=\psi(x) \mathbb{P}-\text { almost everywhere }
$$

and

$$
\lim _{T} \psi_{T}^{(4)}=\psi(x) \mathrm{IP} \text {-almost everywhere }
$$

respectively.

\subsection{Asymptotic normality}

If $Q$ has a finite second moment, then the estimators $\psi_{n}^{(i)}, i=1,2,3$ and $\psi_{T}^{(4)}$ are asymptotically normal. For the definition of their variances we need some notations. Write

$$
R=\sum_{k=0}^{\infty}(1-\psi(0)) \psi(0)^{k} H^{* k}
$$




$$
M=\sum_{k=0}^{\infty}(1-\psi(0))^{2} \psi(0)^{k}(k+1) H^{* k}
$$

Then for $x \geq 0, \psi(x)=R(x, \infty)$, and $M$ is the twofold convolution of $R$,

$$
M=R^{* 2}
$$

For $v \geq 0$ define

$$
g(v)=\int\left(v-(x-u)^{+}\right)^{+} M(d u)
$$

and write $i d$ for the identity on $\mathbb{R}: i d(v)=v, v>0$.

THEOREM : Let $x, c, \lambda>0$ be fixed, and $Q$ a nondegenerate claim amount distribution with finite second moment, such that (3) holds. Then for $i=1,2$ there exist positive numbers $\sigma^{(i)}$ such that the distribution of

$$
n^{1 / 2}\left(\psi_{n}^{(i)}(x)-\psi(x)\right)
$$

converges weakly to $N\left(0, \sigma^{(i)}\right)$. We have

$$
\begin{gathered}
\sigma^{(1)}=K^{2} \operatorname{Var}_{Q}(g-\psi(x) i d) \\
\sigma^{(2)}=K^{2} \operatorname{Var}_{Q}\left(g-Q[g] \mu^{-1} i d\right)
\end{gathered}
$$

where

$$
Q[g]=\int g(v) Q(d v)
$$

In case 4 ), the distribution of

$$
(\lambda T)^{1 / 2}\left(\psi_{T}^{(4)}(x)-\psi(x)\right)
$$

converges weakly to $N\left(0, \sigma^{(4)}\right)$ when $T \rightarrow \infty$, with

$$
\sigma^{(4)}=K^{2} Q(g-\psi(x) i d)^{2}
$$

In case 3 ) let

$$
\sigma^{(3)}=K^{2} \operatorname{Var}_{Q}(g-a \times i d)
$$

where $a=\operatorname{Cov}_{Q}(g, i d) / \operatorname{Var}_{Q}(i d)$. If $\sigma^{(3)}>0$ then the distribution of

$$
n^{1 / 2}\left(\psi_{n}^{(3)}(x)-\psi(x)\right)
$$

converges weakly to $N\left(0, \sigma^{(3)}\right)$. The constant $K$ equals

$$
K=\psi(0) /(\mu(1-\psi(0)))
$$

The proofs for (2.1) and (2.2) are indicated in [6]. The situation $\sigma^{(3)}=0$ occurs, e.g., if $Q(x, \infty)=0$. 


\subsection{Comparison of variances}

We shall give the proof for

a) $\sigma^{(2)}>0$ and

b) $\sigma^{(2)} \leq \sigma^{(1)}$

which are not contained in [6].

a) With $h(y)=g(y)-y Q[g] / \mu$ we have

$$
\begin{aligned}
h^{\prime}(y) & =M(x-y, \infty)-Q[g] / \mu \\
& =M(x-y, \infty)-M * H(x, \infty)
\end{aligned}
$$

which is increasing. This yields convexity of $h$. Since $h^{\prime}$ changes sign and $h(y) \rightarrow \infty, y \rightarrow \infty$, and $h(0)=0, h$ must have exactly two distinct zeroes. Hence

$$
\sigma^{(2)}=\int h^{2}(y) Q(d y)
$$

cannot be zero. We see that $h$ changes sign exactly once.

b) Using the relation

$$
\operatorname{Var}(X)-\operatorname{Var}(Y)=\operatorname{Var}(X-Y)+2 \operatorname{Cov}(X-Y, Y)
$$

we see that it suffices to show that

$$
\operatorname{Cov}_{Q}(k-h, h) \geq 0
$$

with $k(y)=g(y)-\psi(x), y>0$. This relation is equivalent to

$$
\operatorname{Cov}_{Q}(i d, h)(Q[g] / \mu-\psi(x)) \geq 0 .
$$

From

$$
\begin{aligned}
Q[g] / \mu-\psi(x) & =M * H(x, \infty)-R(x, \infty) \\
& =R * R * H(x, \infty)-R(x, \infty) \geq 0
\end{aligned}
$$

we see that our assertion will hold provided

$$
\operatorname{Cov}_{Q}(i d, h) \geq 0
$$

If $t_{0}>0$ is the point at which $h$ changes sign, then

$$
\operatorname{Cov}_{Q}(i d, h)=\int y h(y) Q(d y)=\int\left(y-t_{0}\right) h(y) Q(d y) \geq 0 .
$$

This proves $b$ ).

The relation $\sigma^{(3)} \leq \sigma^{(2)}$ follows from efficiency of $\psi_{n}^{(3)}(x)$ (see [6]). 


\section{MONTE-CARLO SIMULATIONS}

The small sample behaviour of our estimators $\psi_{n}^{(1)}(x)$ and $\psi_{n}^{(2)}(x)$ and the level of the bootstrap confidence intervals were investigated using the Monte-Carlo method. We dealt with claim amount distributions which were Lognormal, Pareto, and Gamma distributions. We shall present the results for three special examples which are representative for all cases that had been investigated.

Example I: The claim amount distribution $Q$ is an exponential distribution with parameter 1.25 , the intensity $\lambda$ of the claims process equals 0.125 , the premium rate $c$ equals 1 , and the initial surplus $x$ equals 1 . The mean of $Q$ is 0.8 , the coefficient of variation $\tau$ is 1 , the exact ruin probability without initial surplus $\psi(0)$ equals 0.1 , and the exact ruin probability $\psi(x)$ equals 0.0325 .

Example II: $Q$ is a lognormal distribution with mean and variance of the corresponding normal distribution equal to -0.569 and 0.694 , respectively; $\lambda=0.0125 ; c=1$, and $x=1$. The mean of $Q$ is $0.80092, \tau=1, \psi(0)=0.01$, and $\psi(x)=0.00254$.

Example III: $Q$ is a Pareto distribution with shape parameter 2.054 and location parameter 0.924 , it has density

$$
x \rightarrow 2.054(0.924)^{2.054} x^{-3.054}, \quad x>0.924
$$

The intensity is $\lambda=0.055 ; c=1$ and $x=1$. The mean of $Q$ is $1.8, \tau=3$, $\psi(0)=0.1$, and $\psi(x)=0.0489$.

In these examples the loading is much larger than in practical situations. However, for smaller loadings the computation time for a Monte-Carlo study will increase drastrically.

The distribution functions $\psi_{n}^{(1)}(x)$ and $\psi_{n}^{(2)}(x)$ are simulated for sample size $n=20$ and with a number of 1000 simulations per case. The broken line is the distribution function of the approximating normal distribution. 


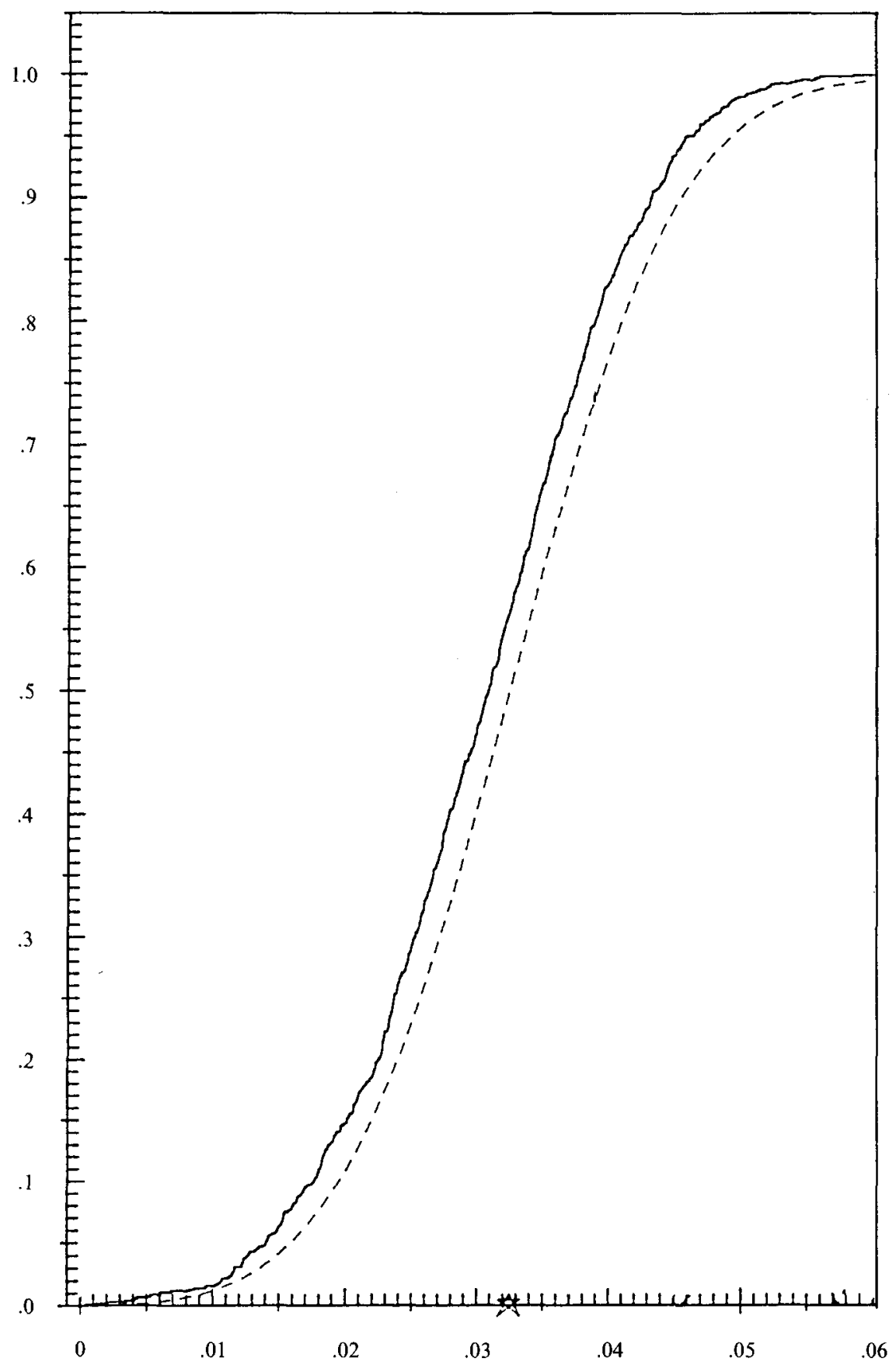

FigURE 1. Distribution function and normal approximation (broken line) for $\psi_{n}^{(2)}(x)$ when $n=20$ in the case of example I (Exponential distribution). 


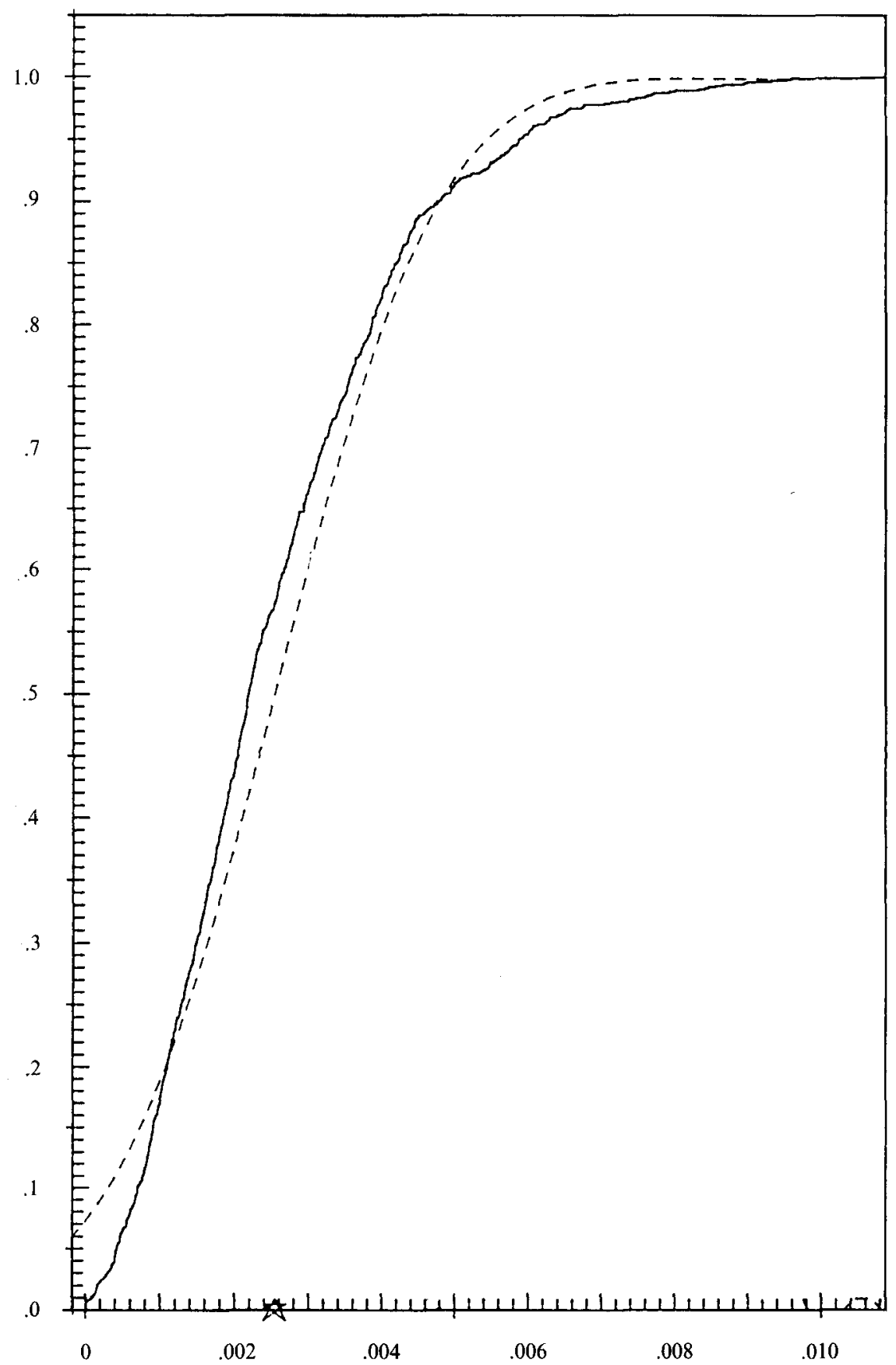

FIGURE 2. Distribution function and normal approximation (broken line) for $\psi_{n}^{(1)}(x)$ when $n=20$ in the case of example II (Lognormal distribution). 


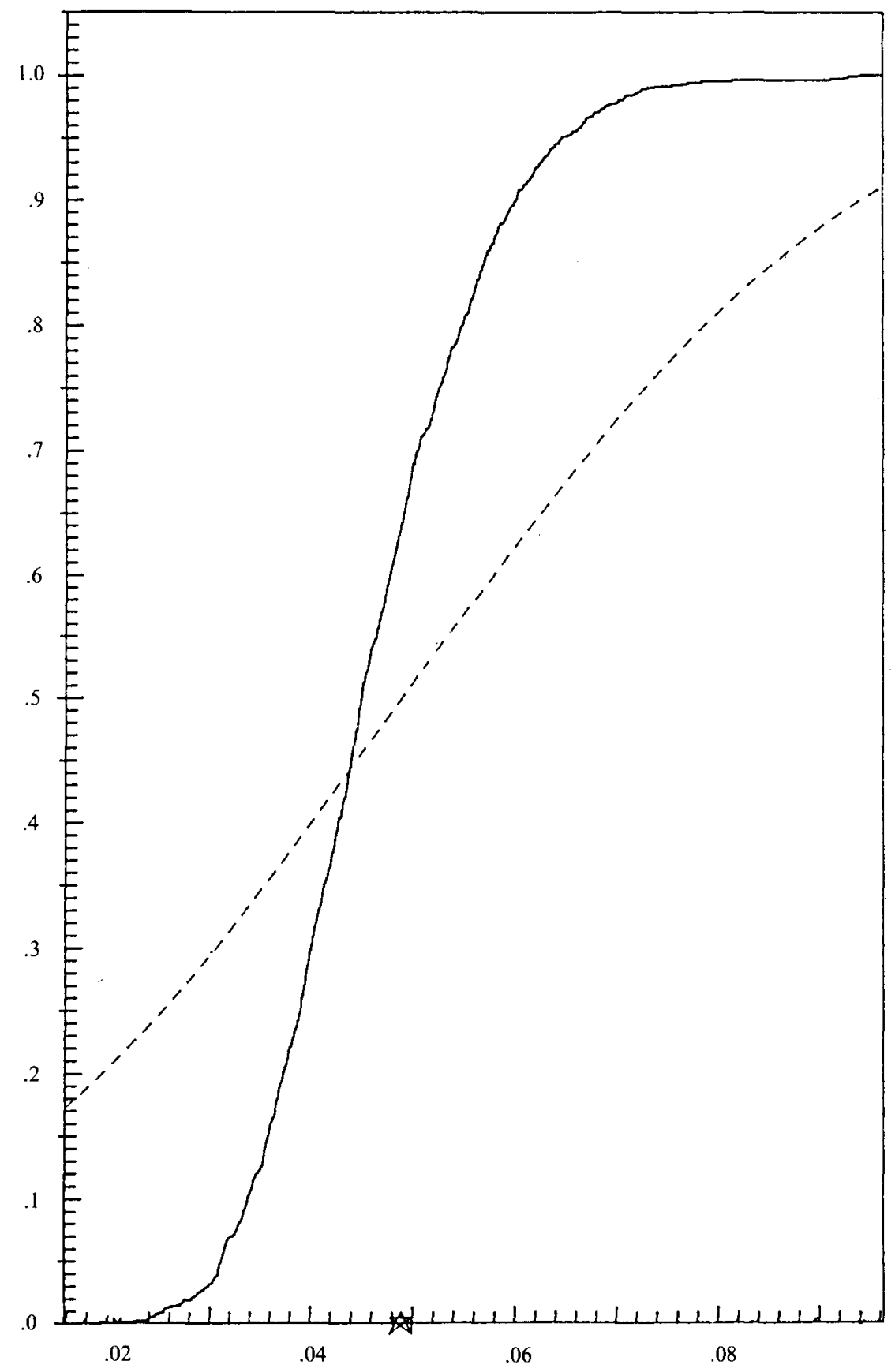

FIGURE 3. Distribution function and normal approximation (broken line) for $\psi_{n}^{(1)}(x)$ when $n=20$ in the case of example III (Pareto distribution). 
In all three cases, the distribution of $\psi_{n}(x)$ is quite well concentrated around the true value $\psi(x)$, but the normal approximation is not at all satisfactory. The above graphs of the distribution function and the normal approximation have been reproduced from [2].

The small sample behaviour of our bootstrap confidence intervals was the following: For sample sizes $n=20$ and $n=100$ we simulated 100 data sets, computed the corresponding confidence interval $C_{n}$ of level $90 \%$ (i.e. $a=0.1$ ) and counted the number of cases in which $\psi(x)$, the true value, was covered by $C_{n}$. Here are our results.

\begin{tabular}{|c|c|c|}
\hline & Sample size & Covers in \% \\
\hline Example I & 20 & 85 \\
$\psi_{n}^{(1)}(x)$ & 100 & 94 \\
Example I & 20 & 76 \\
$\psi_{n}^{(2)}(x)$ & 100 & 88 \\
Example II & 20 & 77 \\
$\psi_{n}^{(1)}(x)$ & 100 & 93 \\
Example II & 20 & 71 \\
$\psi_{n}^{(2)}(x)$ & 100 & 89 \\
Example III & 20 & 78 \\
$\psi_{n}^{(1)}(x)$ & 100 & 89 \\
Example III & 20 & 71 \\
$\psi_{n}^{(2)}(x)$ & 100 & 89 \\
\hline
\end{tabular}

Changing from sample size $n=20$ to $n=100$ did drastically increase covering probability in all cases concerned in our investigation, especially in cases where the covering probability for sample size 20 was small.

\section{ACKNOWLEDGEMENT}

The Monte-Carlo simulations for the investigation of $\psi_{n}^{(1)}(x), \psi_{n}^{(2)}(x)$ and the corresponding confidence intervals based on the normal approximation were done by S. ENGELÄNDER, a student of mine, in connection with his work on the estimation of ruin probabilities in his diploma thesis at the university of Cologne. He proposed the estimator $\psi_{n}^{(2)}(x)$ and computed its asymptotic variance $\sigma^{(2)}$. Mr. ENGELÄNDER'S simulations ran on the CDC 70/76 under Scope 2.1 at the University of Cologne. The simulations for the performance of bootstrap confidence intervals were programmed by Mr. J. STEIN. I tank the Verein zur Förderung der Versicherungswissenschaften in Hamburg e.V. who financed the cooperation with Mr. STEIN. The bootstrap simulations were done on the SIEMENS 7881 under BS 3000 at the University of Hamburg. The revision of the first version of this manuscript was based on remarks of two referees which are gratefully acknowledged. 


\section{REFERENCES}

Bühlmann, H. (1980). Mathematical methods in risk theory. Springer, New York.

ENGELÄNDER, S. (1987). Konfidenzintervalle für empirische Ruinwahrscheinlickeiten: asymptotisches Verhalten und Monte-Carlo-Simulation. Diploma Thesis, University of Cologne.

Feller, W. (1970) An Introduction to Probability Theory and Its Applications, 2, Wiley, New York.

FREES, E.W. (1986). Nonparametric estimation of the probability of ruin. ASTIN Bulletin 16, 8190.

Grandell, J. (1979). Empirical bounds for ruin probabilities. Stoch Process and Their Applications 8 , 243-255.

HIPP, C. (1988). Efficient estimators for ruin probabilities. Proc. 4th Prague Symp. Asympt. Statist.

TAYLOR, G. C. (1985). A heuristic review of some ruin theory results. ASTIN Bulletin 15, 73-88.

\section{Christian Hipp}

Universität Hamburg, Institut für mathematische Stochastik, Bundesstrasse 55, D-2000 Hamburg 13. 